\title{
Application of metasurfaces in the design of performance-enhanced low-profile antennas
}

\author{
Ikmo Park \\ Department of Electrical and Computer Engineering, Ajou University, 206 Worldcup-ro, Youngtong-gu, Suwon 16499, \\ Republic of Korea
}

Received: 17 September 2018 / Accepted: 17 November 2018

\begin{abstract}
This paper presents a review of metasurface-based antennas conducted at the Microwave Communication Laboratory (MCL) of Ajou University in the Republic of Korea. In this paper, profile miniaturization, bandwidth enhancement, multiband operation, and radiation pattern control of metasurfacebased antennas are considered. The paper first presents metasurface-based antennas implemented by placing various radiators on top of the metasurface. It then presents antennas implemented by placing the radiators below the metasurface with and without the ground plane. Metasurface-based antennas are not only able to achieve high efficiency with a low profile but they are also able to generate extra resonances from the metasurface structures, which significantly enhances the overall performance of the antennas. These additional resonances were utilized in multiband and/or wideband operations. In addition, the design of a planar compact wide-gainbandwidth metasurface-based antenna and its radiation characteristics are presented at a terahertz $(\mathrm{THz})$ frequency range. The $\mathrm{THz}$ antennas were designed with metasurfaces and a planar leaky-wave feeding structure. Finally, the outlook on future research at the MCL for antenna-related work and their applications using metasurfaces is provided.
\end{abstract}

Keywords: Antenna / array / artificial magnetic conductor / circular polarization / crossed dipole/ leaky-wave / metamaterial / metasurface / sequential rotation / surface wave resonance / wideband

\section{Introduction}

The electromagnetic properties of materials can be classified into four types: double positive (DPS), epsilon negative (ENG), mu negative (MNG), and double negative (DNG) - depending on the values of permittivity and permeability [1]. Dielectrics, magnetic materials, and plasmas are materials that exist in nature with DPS, MNG, and ENG values, respectively. A material that has negative permittivity and permeability - that is, a material that has a negative refractive index (DNG) - is defined as a metamaterial that typically does not exist in nature and can only be implemented through artificial processing [2]. In 1968, Veselago published the first study on the properties of materials with negative permittivity and permeability simultaneously [3]. These materials are called left-handed materials (LHM) because they have the characteristic of electromagnetic waves that the group velocity and the phase velocity are reversed and propagate in the opposite direction. In 1999, Pendry published a

\footnotetext{
* e-mail: ipark@ajou.ac.kr
}

study on the implementation of a medium with negative permeability using split ring resonators (SRRs) [4]. In 2000, Schultz and coworkers experimentally demonstrated the properties of materials with a negative refractive index using periodically implemented metal lines and SRRs [5,6]. Since then, many studies have been undertaken that have obtained excellent results. The history and development of metamaterials are well documented in reference [7]. However, materials with a negative refractive index have a narrow bandwidth, high dispersion, and anisotropy characteristics, and they are very difficult to implement and apply in real applications because of their high loss.

In addition to DNG materials, researchers are studying metamaterials with other extreme values [8], such as zero-index materials (ZIM), epsilon near zero (ENZ), mu near zero (MNZ), and perfect magnetic conductors (PMC). Permittivity and permeability can be implemented with a positive value for these materials, thereby facilitating fabrication and practical applications. In particular, in 1999, Sievenpiper et al. implemented a PMC with the high-impedance surface (HIS) of a periodic mushroom structure composed of patches and 
vias [9]. Since then, several structures have been found that have properties similar to this HIS structure, namely, reactive impedance surfaces and electromagnetic bandgap (EBG) structures; these structures are generally referred to as artificial magnetic conductors (AMC). Thus, many metamaterials can be defined as materials in new or artificial structures that have been designed to have properties that are not present or difficult to obtain in nature. Unlike perfect electric conductor (PEC), AMCs have characteristics whereby phase change does not occur when the electromagnetic wave is vertically incident and reflected. The current flowing in the conductor on the AMC is in the same direction as the imaging current formed in the AMC. Such a physical phenomenon can improve the antenna characteristics due to constructive interference, even when the antenna is positioned very close to the AMC, which can be very useful for the miniaturization of the antenna and improvement of its characteristics [10]. An AMC structure that has recently attracted attention is a metasurface structure that implements AMC characteristics through a periodic surface that consists of a single layer [11]. The metasurface structure is realized in two dimensions and thus requires a small physical space; this is in contrast to general metamaterials, which have a three-dimensional structure. These properties open the door to the development of new theory and applications [12], and many excellent review articles on the fundamentals and applications of metasurfaces can be found [13-22]. Because AMCs are typically constructed in a two-dimensional structure and they are included in a subset of metasurfaces [14], these AMCs are termed as the metasurface throughout this paper.

A metasurface, which is a two-dimensional equivalent of a metamaterial, is essentially a surface distribution of electrically small scatterers that can manipulate and control electromagnetic waves $[11,14,20-22]$. The functionality of the metasurfaces is governed by the specific geometrical shape of the scatterers that are fabricated on the dielectric substrate [23]. Metasurfaces can provide the novel characteristics of an ultra-low profile and enhanced performance in gain, radiation pattern, and bandwidth $[24,25]$. These metasurfaces have many other inherent advantages, such as low cost, low mass, extreme flatness, and easy fabrication, which have led to metasurface research expanding from microwave to optical frequencies [11], and many extraordinary properties of metasurface antennas have been demonstrated [26-29]. In this paper, an attempt is made to review some of the applications of antennas designed using metasurfaces at the Microwave Communication Laboratory (MCL) of Ajou University. One major concern regarding antennas that use a metasurface is the existence, due to the presence of a finite-size metasurface structure, of resonances that are additional to the conventional resonance created in the main radiating element. Based on the characteristics, various metasurface-based low-profile antennas that perform much better than conventional antennas were designed and investigated.

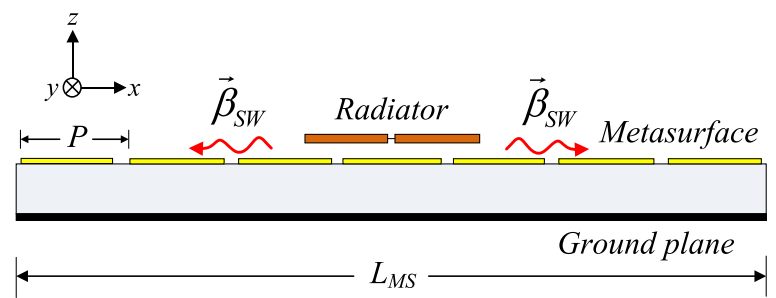

(a)

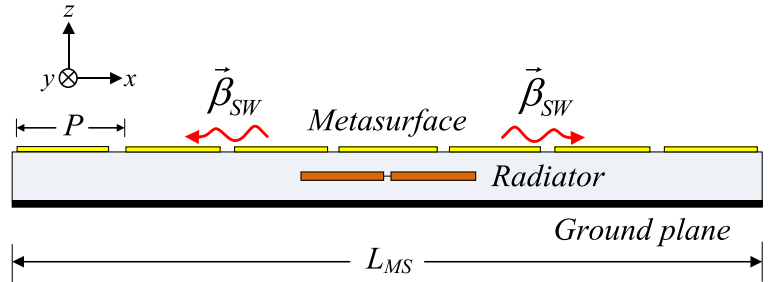

(b)

Fig. 1. Surface waves propagating on the metasurface-based antenna: The driven radiator is placed (a) above the metasurface structure and (b) sandwiched between the metasurface and the ground plane.

\section{Cavity model for surface wave resonances}

Any primary radiation elements, such as horizontal dipoles and microstrip patch antennas, that are based on a finitesized metasurface structure yield extra resonances for the radiation structures [30,31]. These extra resonances are caused by surface waves propagating on the metasurface structure, as shown in Figure 1. The driven radiator can be placed above the metasurface structure or sandwiched between the metasurface and the ground plane. At resonances, the total length of the metasurface structure is equal to the resonant length for a given surface wave that travels along the metasurface. Therefore, for an antenna structure, different waves generate different additional resonances. A detailed description of the cavity model for the surface wave resonances can be found in references [32,33].

By considering the finite-sized metasurface structures as a cavity, the surface wave resonances on a finite metasurface-based antenna can be qualitatively determined by the following equation:

$$
\beta_{\mathrm{SW}} \times L_{\mathrm{MS}}=\pi,
$$

where $\beta_{\mathrm{SW}}$ represents the propagation constant of the surface wave resonances, and $L_{\mathrm{MS}}$ is the total length of the metasurface structure given by

$$
L_{\mathrm{MS}}=N \times P,
$$

where $N$ represents the number of cells, and $P$ is the periodicity of the metasurface. Inserting equation (2) into equation (1), we have

$$
\beta_{\mathrm{SW}}=\frac{\pi}{N \times P} .
$$




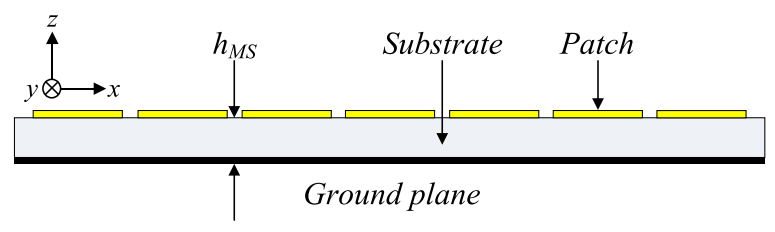

(a)

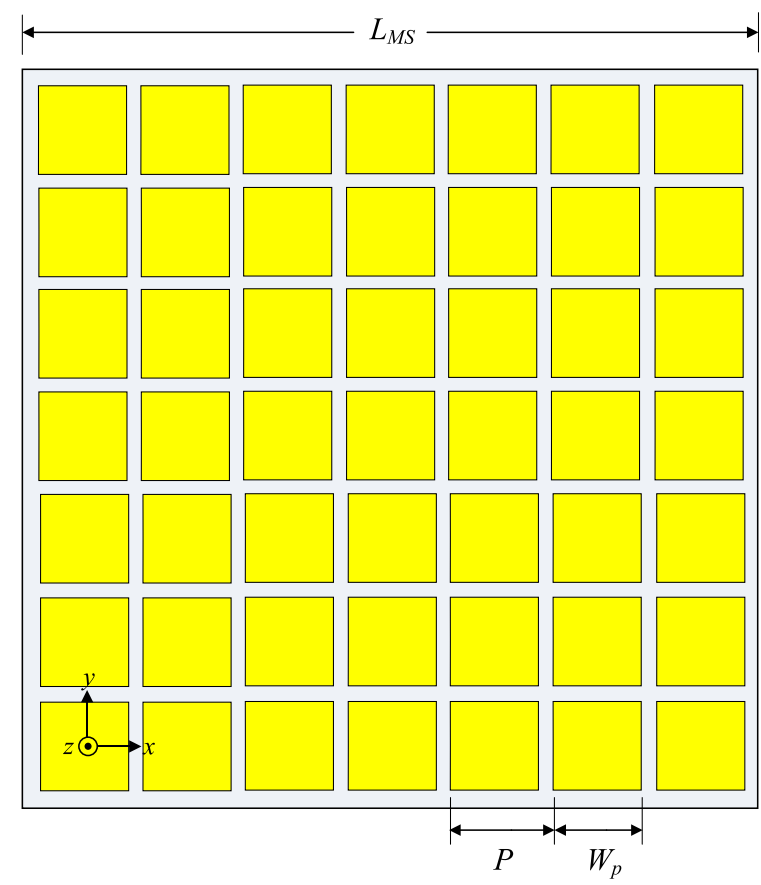

(b)

Fig. 2. Geometry of the general metasurface structure: (a) side view and (b) top view. The metasurface structure periodically arranged with square metal patches on a grounded substrate. Its periodicity is $P$ and the size of the metallic patch is $W_{\mathrm{p}}$.

It was shown in reference [9] that the propagating constant of the surface waves traveling and decaying away from the metasurface is related to the decay constant $(\alpha)$ and the frequency $(\omega)$ by the following expression:

$$
\beta_{\mathrm{SW}}=\sqrt{\eta^{2} \omega^{2}+\alpha^{2}} .
$$

The propagation constant for the transverse magnetic (TM) and transverse electric (TE) waves can be expressed, respectively, as follows:

$$
\begin{aligned}
& \beta_{\mathrm{TM}}=\frac{\omega}{c} \sqrt{1-\frac{Z_{s}^{2}}{\eta^{2}}} \\
& \beta_{\mathrm{TE}}=\frac{\omega}{c} \sqrt{1-\frac{\eta^{2}}{Z_{s}^{2}}}
\end{aligned}
$$

where $\beta_{\mathrm{TM}}$ and $\beta_{\mathrm{TE}}$ represent the propagation constant of the TM and TE waves, respectively, $c$ is the speed of light in

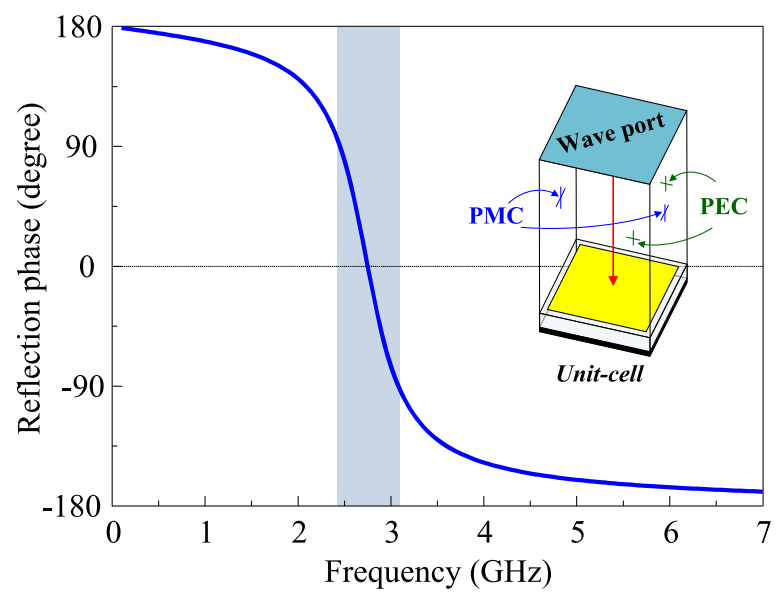

(a)

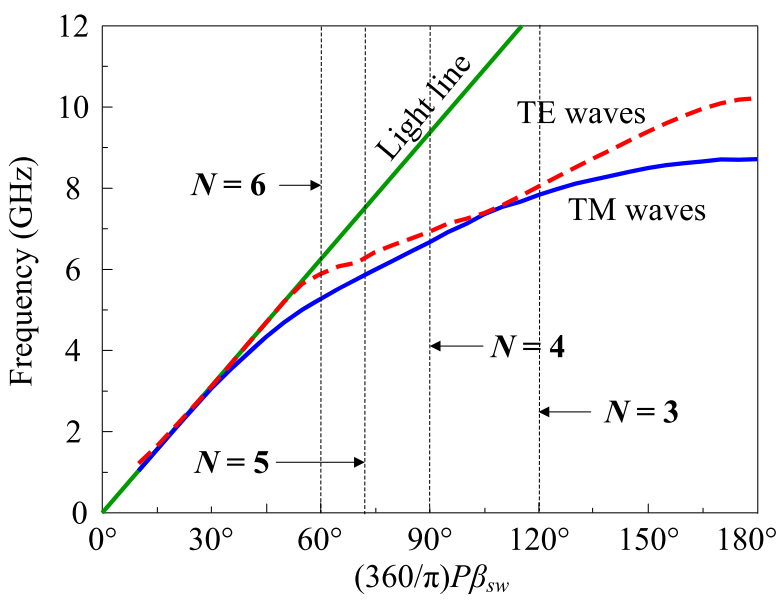

(b)

Fig. 3. Simulation results for the metasurface structure: (a) reflection phase and (b) dispersion diagram.

a vacuum, $\eta$ is the intrinsic impedance, and $Z_{\mathrm{s}}$ is the surface impedance of the metasurface structure.

Figure 2 shows the metasurface structure, which is periodically arranged with square metal patches on a grounded substrate. Its periodicity is $P$, and the size of the metallic patch is $W_{\mathrm{p}}$. As an example of the prediction of the surface wave resonances, RO4003 (Rogers Corporation) was chosen as the substrate for the metasurface structure with a relative permittivity of 3.38 , a loss tangent of 0.0027 and a thickness of $h_{\mathrm{MS}}=4.064 \mathrm{~mm}$. Other design parameters are $P=15 \mathrm{~mm}$ and $W_{\mathrm{p}}=14.4 \mathrm{~mm}$.

A simple model based on a simulation of the scattering parameters of a single-port air-filled waveguide with two PEC and two PMC walls was used to simulate the design, as shown in the inset of Figure 3a [34]. The reflection phase of the metasurface was calculated, as illustrated in Figure $3 \mathrm{a}$. The resonance frequency for the $0^{\circ}$ reflection phase was $2.91 \mathrm{GHz}$, and the $\pm 90^{\circ}$ reflection phase bandwidth was $2.57-3.29 \mathrm{GHz}$. Dispersion diagrams of the metasurface structure were obtained with linked boundary conditions using an ANSYS high-frequency 


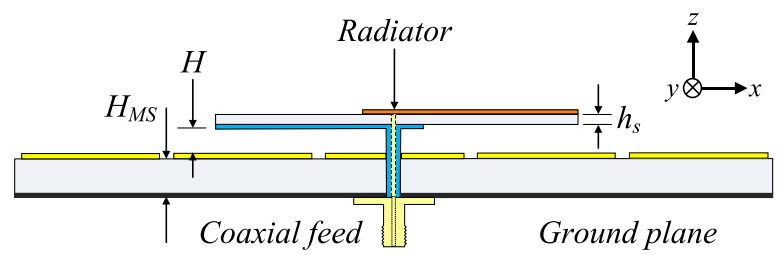

Fig. 4. Basic geometry of the antenna with a radiator over a metasurface structure.

structure simulator [35] and are illustrated in Figure 3b. The first three eigenmodes were calculated and are presented in Figure 3b. The first eigenmode is the TM waves, the second eigenmode is the TE waves, and the third eigenmode is the higher order waves. The resonances were obtained graphically, with the intersection between the dispersion curves and the vertical lines representing the quantity on the right side of equation (1). It was shown that the surface wave resonances on a metasurface-based antenna are mainly determined by the size of the metasurface structure. With the chosen metasurface structure, the dispersion curves for the TM and TE waves are relatively close within the frequency range of interest. Thus, in some cases, there was some overlapping in the resonances generated by these two waves [33].

\section{Application of metasurfaces in antenna design}

\subsection{Antennas with radiators above the metasurface}

The main problem associated with a planar antenna positioned above a metallic reflector is that a quarterwavelength space is required between the radiating elements and the metallic reflector to obtain optimal antenna characteristics. To overcome this problem, a metasurface can be used instead of a metallic reflector as shown in Figure 4. The metasurface, which generally consists of a lattice of metal plates on a grounded dielectric substrate, can mimic a PMC over a certain frequency band $[36,37]$. Consequently, the metasurface allows close placement of the antenna with good impedance matching and a unidirectional radiation pattern. By combining various types of radiators and metasurface structures, lowprofile multiband or wideband antennas with good antenna performance characteristics can be implemented. More interestingly, surface waves propagating on the metasurface structure are excited and generate extra resonances for the radiating system. It was shown that these extra resonances could be favorably used to enhance the bandwidth of the antenna. Various designs of antennas using theses extra resonances are reviewed in this section.

\subsubsection{Dual-band antenna with a single-band CP radiator over metasurface structure}

A dual-band circularly polarized $(\mathrm{CP})$ antenna by placing a single-band CP crossed-dipole radiator on a finite metasurface structure was presented in reference [38]. Dual-band operation was obtained by utilizing the original band of the

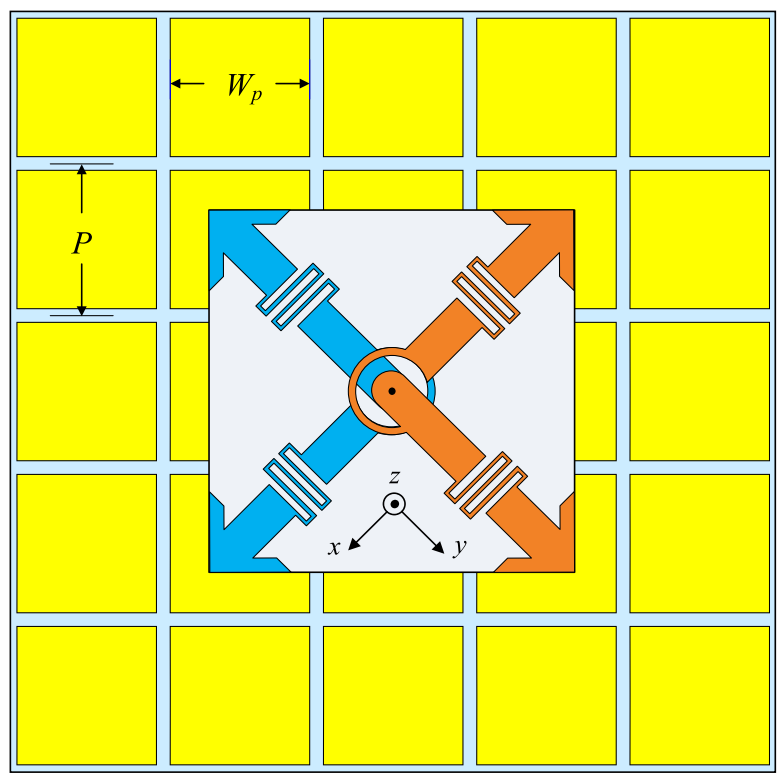

(a)

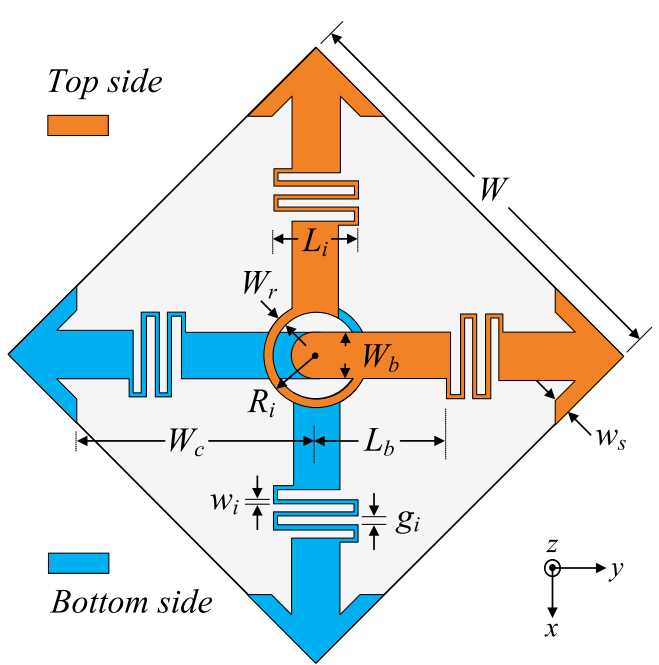

(b)

Fig. 5. Geometry of the low-profile CP crossed dipole on the metasurface for dual-band operation: (a) top view of the metasurface-integrated antenna and (b) top view of the singleband arrowhead-shaped crossed-dipole radiator.

crossed dipole radiator and the first extra resonance caused by the surface waves propagating on the finite metasurface structure; the original band is the lower one, while the additional band is the upper one, which is mainly controlled by the size of the metasurface structure. The dual-band antenna configuration is shown in Figure 5. It consists of a compact arrowhead-shaped crossed dipole [39] above a $5 \times 5$ metasurface. The antenna was optimized for the GPS L1/L2 bands with low-profile, broadband characteristics and excellent $\mathrm{CP}$ radiation. The final design, with an overall size of $85 \times 85 \times 11.493 \mathrm{~mm}^{3}$ (approximately $0.3485 \times 0.3485 \times 0.047 \lambda_{o}^{3}$ at $1.23 \mathrm{GHz}$ ), yields a measured $-10 \mathrm{~dB}$ impedance bandwidth of 


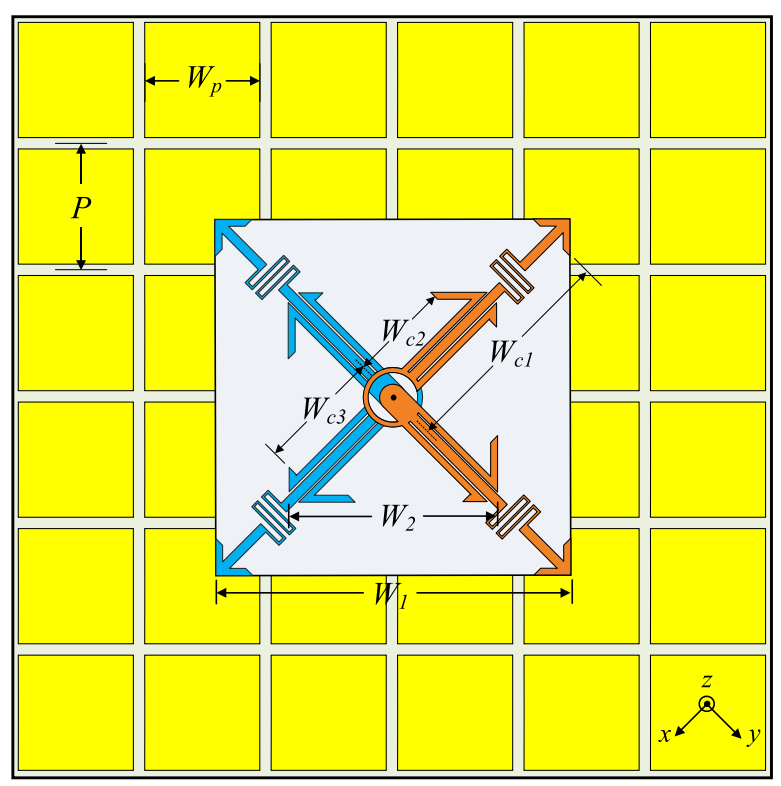

(a)

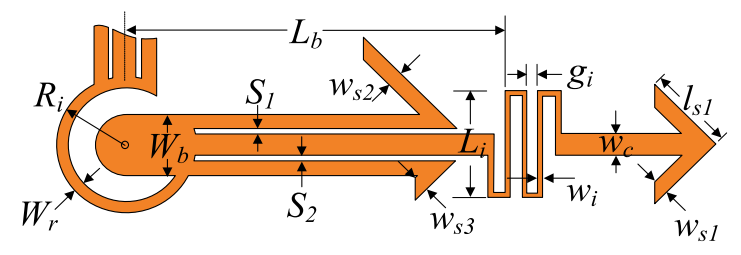

(b)

Fig. 6. Geometry of the crossed trident-shaped dipole element over a metasurface structure for multi-band operation: (a) top view of the metasurface-integrated antenna and (b) top view of a trident arm with a vacant-quarter printed ring.

1.202-1.706 GHz and the $3-\mathrm{dB}$ axial ratio (AR) bandwidths of $1.205-1.240 \mathrm{GHz}$ and $1.530-1.625 \mathrm{GHz}$. The proposed antenna exhibits right-hand circular polarization (RHCP) in both bands and yielded gains of 5.60 and $5.31 \mathrm{dBic}$ and radiation efficiencies of 95.0 and $96.5 \%$ at the GPS L1/L2 bands, respectively. Based on the proposed design method, the antenna volume can be significantly decreased by using high-permittivity materials for the metasurface substrate. This was also demonstrated in reference [38] with a dualband design that used $\varepsilon_{\mathrm{r}}=20$.

\subsubsection{Multiband antenna with multibranch CP radiator over metasurface structure}

Several wireless communication systems use multiple frequency bands that have a large frequency ratio to improve throughput [40]. For example, WLAN uses a lower frequency band of $2.4-2.485 \mathrm{GHz}$ for the IEEE $802.11 \mathrm{~b} / \mathrm{g}$ standard and two upper frequency bands of 5.150-5.350 and $5.725-5.875 \mathrm{GHz}$ for the IEEE 802.11a standard. The basic crossed multibranch dipole with a single-feed structure can be easily modified for use in these systems. As an example, a crossed trident-shaped dipole was optimized to have $\mathrm{CP}$ radiation at the $2.4,5.2$, and
$5.8 \mathrm{GHz}$ WLAN bands [41]. As shown in Figure 6, the triband-crossed dipoles are backed by a finite metasurface structure to achieve low-profile, broadband characteristics and a unidirectional radiation pattern at three bands. The metasurface structure was designed to act as a PMC over the $2.4 \mathrm{GHz}$ band while performing like a PEC over the 5.2 and $5.8 \mathrm{GHz}$ bands. In addition, the extra resonance generated by the surface waves propagating on the metasurface structure was properly utilized to produce an improvement of the $3-\mathrm{dB}$ AR bandwidth at the $2.4 \mathrm{GHz}$ band. The fabricated prototype has an overall size of approximately $0.576 \times 0.576 \times 0.14 \lambda_{o}^{3}\left(\lambda_{\mathrm{o}}\right.$ is the free space wavelength at $2.4 \mathrm{GHz})$. At $2.4 \mathrm{GHz}$, it has a $-10 \mathrm{~dB}$ impedance bandwidth of $2.21-2.62 \mathrm{GHz}, 5.02-5.44 \mathrm{GHz}$, and $5.62-5.96 \mathrm{GHz}$, and a $3-\mathrm{dB}$ AR bandwidth of $2.34-$ $2.58 \mathrm{GHz}, 5.14-5.38 \mathrm{GHz}$, and $5.72-5.88 \mathrm{GHz}$. In addition, the antenna system has a unidirectional RHCP radiation pattern, high radiation efficiency, and stable operation over all three bands.

\subsubsection{Dual-band antenna over dual-band metasurface structure}

A novel miniaturized dual-band metasurface structure was utilized for dual-band operation. A metasurface unit cell that employs four slots in the metallic patch was used to achieve miniaturization as well as easy control of the second/first resonant frequency ratio by simply changing the slot shape for a given metallic patch size $[42,43]$. A dual-band metasurface structure was employed as a reflector to achieve a low profile and broadband characteristics for dual-band operation with a large frequency ratio [42]. Figure 7 shows the geometry of a single-feed lowprofile dual-band crossed asymmetric dipole antenna. The radiator was designed to produce $\mathrm{CP}$ radiation at the 2.4 and $5.2 \mathrm{GHz}$ bands. To match the operating frequencies of the radiator, the first and second resonances of the metasurface structure were adjusted by using four Tshaped slits in each of the unit-cell patches, as shown in Figure 7c. More importantly, the extra resonances caused by the surface waves on the finite metasurface structure were generated and were consequently utilized to improve the 3-dB AR bandwidths at both bands. The final design, with an overall size of $0.5760 \times 0.576 \times 0.088 \lambda_{0}^{3}$ at $2.4 \mathrm{GHz}$, results in a $-10 \mathrm{~dB}$ impedance bandwidths of 2.20 $2.60 \mathrm{GHz}$ and $4.90-5.50 \mathrm{GHz}$, and $3-\mathrm{dB}$ AR bandwidths of $2.30-2.50 \mathrm{GHz}$ and $5.05-5.35 \mathrm{GHz}$, as well as a highly efficient unidirectional radiation pattern. This antenna is a good candidate for dual-band wireless communications, such as WLAN, RFID, and WiMAX applications.

Another dual-band antenna composed of a wideband planar monopole horizontally suspended over the dualband metasurface structure was designed and tested [43]. The measurements resulted in a good matching condition for the dual-band antenna. Both PEC and PMC surfaces are known to have a strong coupling with the antennas above them [37]. When the antenna is placed in close proximity and parallel to a PEC, its impedance matching is rather difficult. In contrast, the impedance matching of an antenna in close proximity to a PMC appears to be easier. Accordingly, the antenna system was designed to prove 


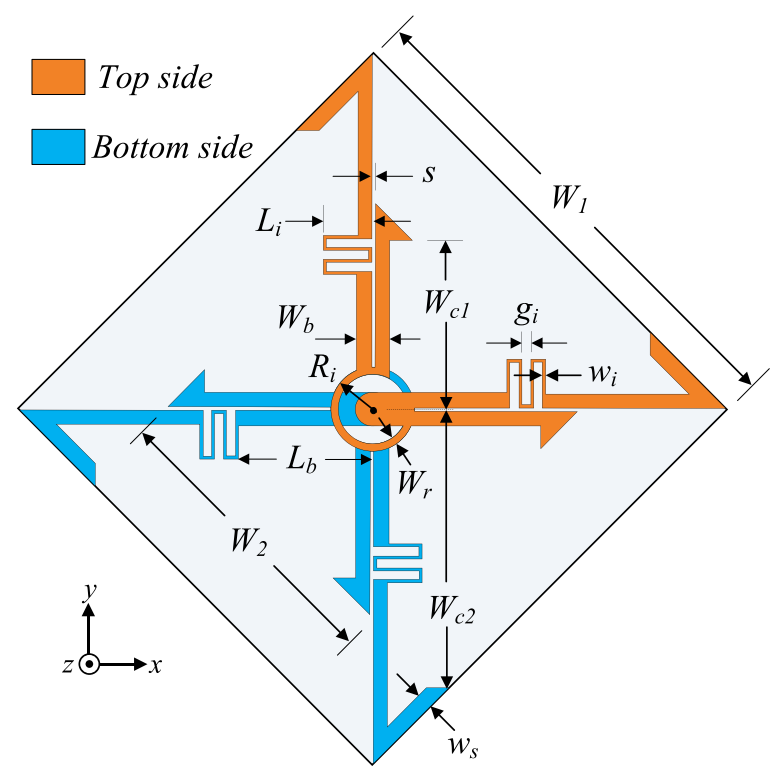

(a)
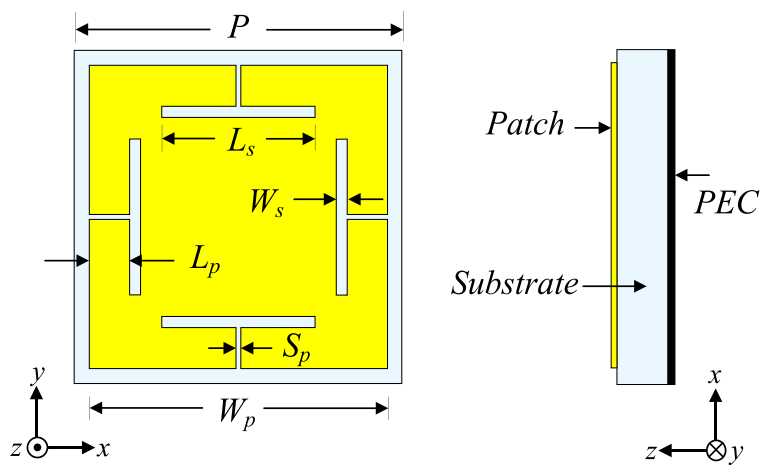

(b)

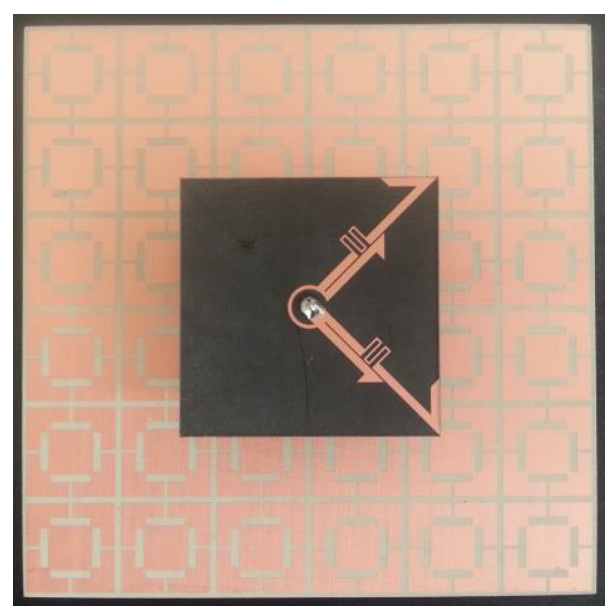

(c)

Fig. 7. Geometry of the dual-band antenna over the dual-band metasurface structure: (a) top view of a crossed asymmetric dipole radiator, (b) unit-cell of the dual-band metasurface structure, and (c) photograph of the fabricated antenna.

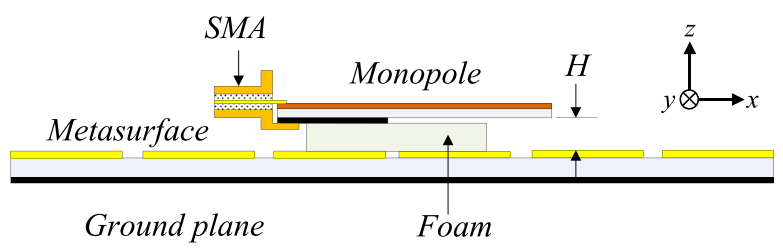

(a)

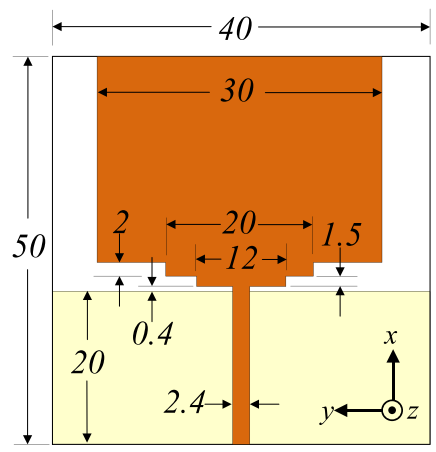

(b)

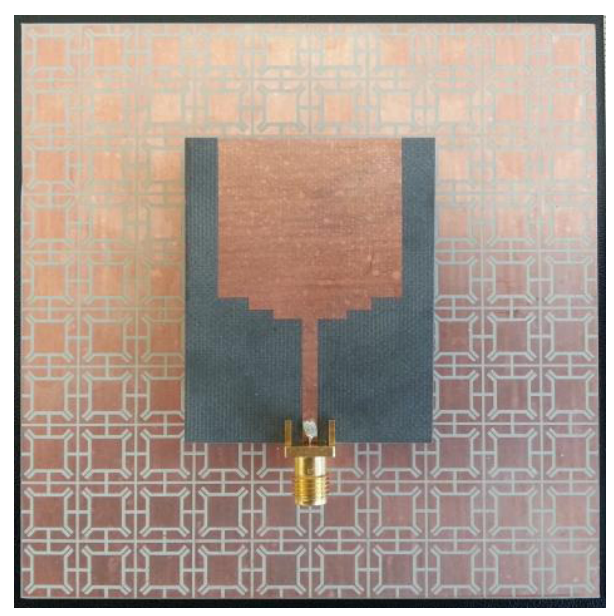

(c)

Fig. 8. Geometry of the dual-band antenna over the dual-band metasurface structure: (a) side view of the antenna, (b) top view of an ultra-wideband monopole, and (c) photograph of the fabricated antenna.

that the proposed metasurface structure worked as a PMC at the resonance frequencies. We confirmed the features of the proposed metasurface structure shown in Figure 8 by designing, fabricating, and measuring a dual-band antenna. A $10 \times 10$ cell array was chosen to form a metasurface structure with an overall size of $100 \times 100 \times 1.27 \mathrm{~mm}^{3}$. A wideband monopole antenna was suspended $6 \mathrm{~mm}$ above the metasurface using Rohacell structural foam having $\varepsilon_{\mathrm{r}}=1.07$ and $\tan \delta=0.0006$. A wideband monopole that was fed by a $50 \Omega$ microstrip line was fabricated on both sides of an RT/Duroid 5880 substrate having $\varepsilon_{\mathrm{r}}=2.2$, tan $\delta=0.0009$, and thickness $=0.7874 \mathrm{~mm}$. The final design, with an overall size of $0.8 \times 0.8 \times 0.06 \lambda_{o}^{3}$ at $2.4 \mathrm{GHz}$, yielded measured impedance bandwidths of $2.37-2.59 \mathrm{GHz}$ and 


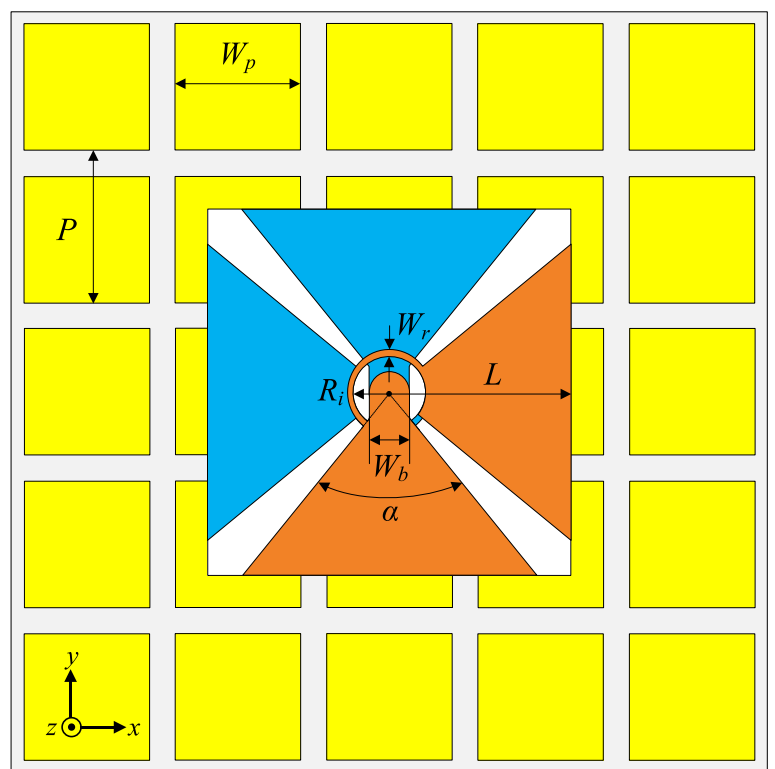

Fig. 9. Geometry of a crossed triangular bowtie dipole antenna over a metasurface structure for a wideband CP operation.

4.28-5.62 GHz, while the simulated impedance bandwidths were $2.40-2.58 \mathrm{GHz}$ and $4.37-5.50 \mathrm{GHz}$. The first and second bands of the antenna system verified the dual-band capability of the proposed metasurface structure. The impedance bandwidth became narrower after loading the antenna over the metasurface because the impedance bandwidth of the antenna system depended on the operation bandwidth of the metasurface structure.

\subsubsection{Wideband antenna on a metasurface structure}

A wideband $\mathrm{CP}$ antenna with a metasurface structure was presented in references $[44,45]$ based on design principle that was similar to those described in the first two previous design examples. The primary radiation elements are composed of two bowtie dipoles that are arranged orthogonally through double-printed rings to allow easy impedance matching and produce good CP radiation, as shown in Figure 9. Planar periodic square metallic plates, which behave as a metasurface structure when placed on a grounded dielectric substrate, are incorporated with crossed bowtie dipoles to achieve the desired unidirectional radiation, a low profile, and wideband features. The final design, with an overall size of $0.75 \times 0.75 \times 0.11 \lambda_{o}^{3}\left(\lambda_{\circ}\right.$ is the free space wavelength at $5 \mathrm{GHz}$ ), resulted in a measured $-10 \mathrm{~dB}$ impedance bandwidth of $54.2 \%$ $(4.3-7.5 \mathrm{GHz})$ and a $3-\mathrm{dB}$ axial ratio bandwidth of $33.3 \%(5-7 \mathrm{GHz})$. The antenna yields an RHCP and broadside gain of approximately $7.9 \mathrm{dBic}$ across the $\mathrm{CP}$ operating bandwidth.

A dual-wideband CP antenna was also presented based on the strategy of combining multimode resonances [45]. The primary radiators were two barb-shaped dipoles and two bowtie dipoles printed on the same substrate as shown in Figure 10. They were placed over a metasurface

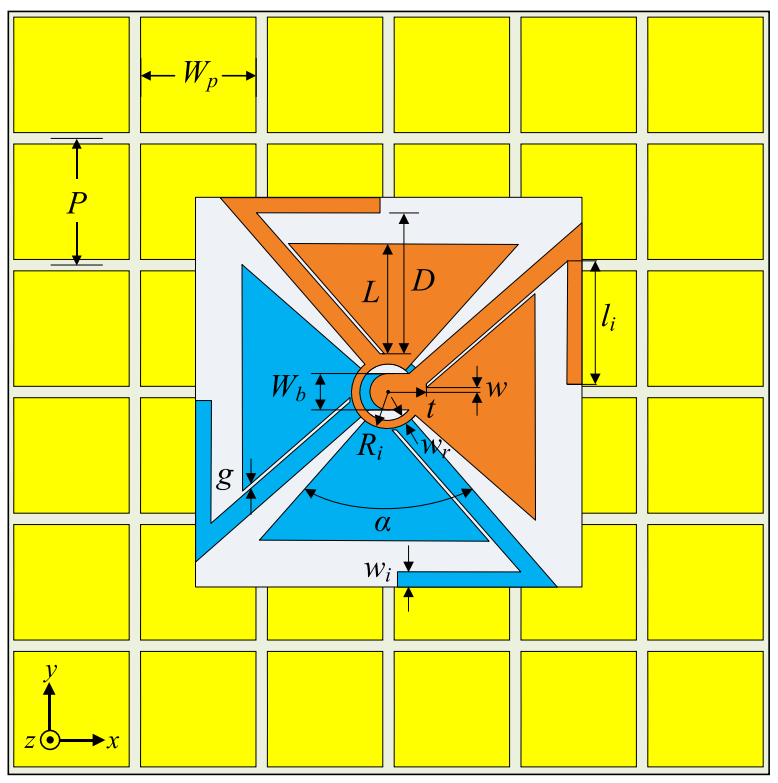

Fig. 10. Geometry of a crossed triangular bowtie with a barbshaped dipole antenna over a metasurface structure for dual wideband $\mathrm{CP}$ operation.

for the purpose of low-profile wideband antenna design. Dual-wideband operation can be achieved by properly combining the fundamental mode of the barb-shaped dipole, the bowtie mode, the slot mode, the second mode of a barb-shaped dipole, and the extra resonance generated by the metasurface structure. The metasurface structure was designed to act as a PMC over the lower band and perform like a PEC over the upper bands, as in the case of reference [41]. The metasurface consisted of $6 \times 6$ square unit cells, which were printed periodically on a $72 \times$ $72 \mathrm{~mm}^{2}, 3.175 \mathrm{~mm}$ thick Cer-10 substrate with a dielectric constant of 10.2 and a loss tangent of 0.0035 . The final design, with an overall size of $0.64 \times 0.64 \times 0.16 \lambda_{o}^{3}$ at $2.4 \mathrm{GHz}$, had a measured impedance bandwidth of $40 \%$ $(2-3 \mathrm{GHz})$ and $49.5 \%(3.8-6.3 \mathrm{GHz})$ for the lower band and upper bands, respectively. The measured 3-dB AR bandwidths were $19.3 \%(2.25-2.73 \mathrm{GHz})$ and $33.8 \%$ $(4.3-6.05 \mathrm{GHz})$ for the lower band and upper bands, respectively. The antenna performed an average gain of about $6.6 \mathrm{dBic}$ across the low $\mathrm{CP}$ region and $7.4 \mathrm{dBic}$ across the high CP region.

\subsection{Antennas with radiators embedded within the metasurface structure}

Most of the antennas investigated above have a relatively large antenna height because their radiating elements are placed above the metasurface structure. Recently, antennas composed of radiators underneath the metasurface have received much attention owing to their short antenna height, broad bandwidth, high efficiency, polarization conversion, and reconfigurable polarization [46-51]. In this section, antennas with radiators placed between the metasurface and the ground plane are presented. 


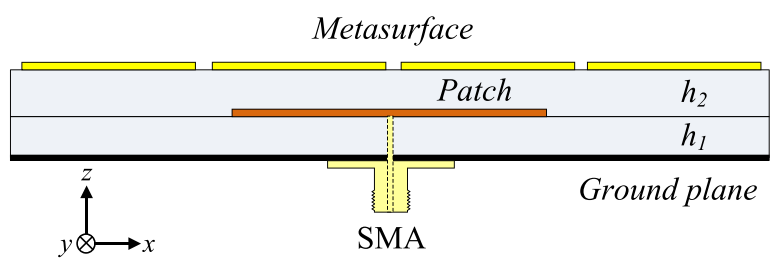

(a)

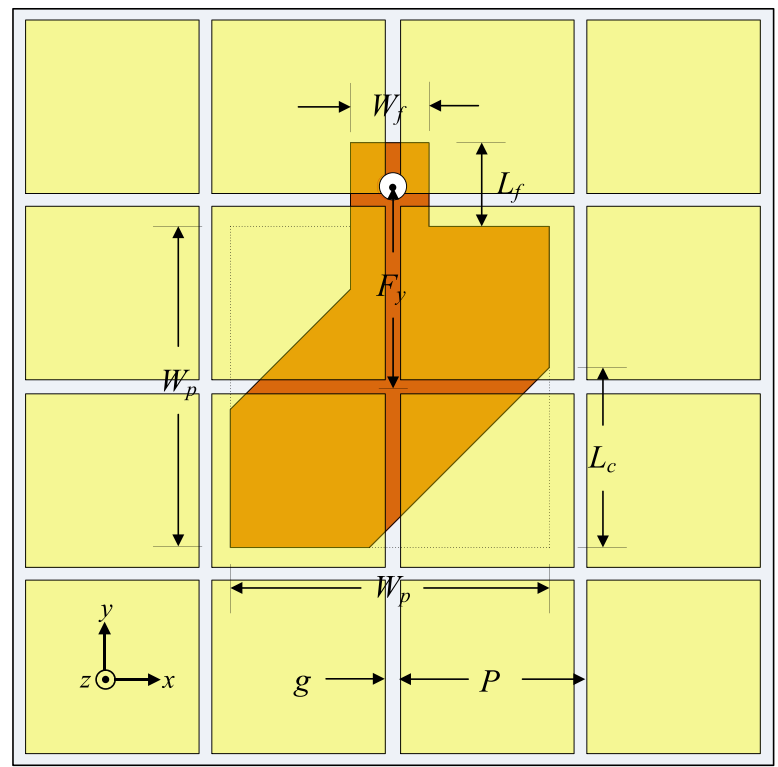

(b)

Fig. 11. Geometry of the antenna: (a) side view and (b) top view. The driven patch is sandwiched between a metasurface and the ground plane. An extended strip with the dimensions $W_{\mathrm{f}} \times L_{\mathrm{f}}$ is added to the driven patch to improve impedance matching.

\subsubsection{Single-feed CP patch antenna}

A low-profile single-feed $\mathrm{CP}$ patch antenna using a metasurface is presented for broadband operation in reference [52]. Figure 11 illustrates the geometry of the antenna, which is composed of a driven patch sandwiched between the metasurface and the ground plane. The driven patch is a truncated corner square patch with the dimensions $W_{\mathrm{p}} \times W_{\mathrm{p}}$ and a truncation of $L_{\mathrm{c}}$. An extended strip with the dimensions $W_{\mathrm{f}} \times L_{\mathrm{f}}$ is added into the driven patch to improve the impedance matching. The antenna comprises a truncated corner square patch sandwiched between a lattice of $4 \times 4$ periodic metal plates and the ground plane. Surface waves propagating on the metasurface are excited. This phenomenon generates additional resonances and minimum $\mathrm{AR}$ points for the radiating structure, consequently broadening the impedance and AR bandwidths of the antenna. The final prototype, with an overall size of $32 \times 32 \times 3 \mathrm{~mm}^{3}\left(0.58 \times 0.58 \times 0.056 \lambda_{o}^{3}\right.$ at $5.5 \mathrm{GHz}$ ), was fabricated and tested. The measurements resulted in a $-10 \mathrm{~dB}$ impedance bandwidth of 4.70 $7.48 \mathrm{GHz}(45.6 \%)$, and a $3-\mathrm{dB}$ AR bandwidth of 4.9 $6.2 \mathrm{GHz}(23.4 \%)$. In addition, the antenna yielded good broadside RHCP radiation with a small gain variation
$(7.0-7.6 \mathrm{dBic})$ and a high radiation efficiency $(>90 \%)$ within the operational bandwidth. The presented antenna has many advantages, including a low profile, broad impedance-matching and 3-dB AR bandwidths, a stable radiation pattern, high gain, and high radiation efficiency.

\subsubsection{CP patch antenna loaded with a solar cell metasurface}

Antennas integrated with solar cells have received much attention along with the rapid development of autonomous communication systems because such a combination could save valuable installation area and reduce design costs. The primary challenge is that the antennas should not block the solar cells from sunlight, and the effectiveness of antennas should not be reduced by the presence of the solar cells. A $\mathrm{CP}$ patch antenna loaded with a metasurface, which was realized with a lattice of $4 \times 4$ square solar cells, was presented in reference [53] to meet those requirements. Figure 12 shows the geometry of the solar cell metasurfaceintegrated antenna. The antenna was composed of a slotted circular patch sandwiched between the metasurface and the ground plane. This arrangement not only allows for the total exposure of the solar cells to sunlight but also improves antenna performance greatly. The final design, with an overall size of $40 \times 40 \times 3.5 \mathrm{~mm}^{3}(0.87 \times$ $0.87 \times 0.076 \lambda_{o}^{3}$ at $6.5 \mathrm{GHz}$ ), yields a $-10 \mathrm{~dB}$ impedance bandwidth of $6.08-7.29 \mathrm{GHz}$ and 3-dB AR bandwidth of 6.0-7.05 GHz. The antenna yields good broadside RHCP radiation with a peak gain of $9.0 \mathrm{dBic}$ and high radiation efficiency of $>90 \%$ across its 3 -dB AR bandwidth. With the promising features of no shading, a planar structure, broad impedance and $\mathrm{CP}$ radiation bandwidths, high gain, and high radiation efficiency, the proposed antenna is a good candidate for autonomous communication systems, as well as CubeSat applications.

\subsubsection{Wideband metasurface-based CP array antenna}

A compact wideband $\mathrm{CP}$ antenna array, which is a set of $2 \times 2$ metasurface-based, $\mathrm{CP}$ patch antennas fed by a sequential-phase (SP) network, was investigated in reference [54]. Figure 13 shows the geometry of the antenna array, which consists of a set of $2 \times 2$ metasurface-based patch antennas fed by an SP network. The single element is composed of a truncated corner square patch sandwiched between the ground plane and the metasurface of a lattice of $4 \times 4$ periodic metal plates [52]. These metasurface-based antennas are incorporated with the SP network of a sequentially rotated series-parallel feed to achieve wideband operation. The radiation pattern and operational bandwidth in the high-frequency region were improved by reducing the spacing between the driven patches while maintaining the structure and overall size of the metasurface. The final design, with an overall size of $64 \times 64 \times$ $2.34 \mathrm{~mm}^{3}$ (approximately $1.26 \times 1.26 \times 0.046 \lambda_{o}^{3}$ at $5.9 \mathrm{GHz}$ ), was fabricated and measured. The antenna array has a measured $-10 \mathrm{~dB}$ impedance bandwidth of $4.40-8.00 \mathrm{GHz}(58.06 \%$ ), a $3-\mathrm{dB}$ AR bandwidth of $4.75-7.25 \mathrm{GHz}(41.67 \%)$, a $3-\mathrm{dB}$ gain bandwidth of $4.8-7.0 \mathrm{GHz}(37.3 \%)$, and a peak gain of $12.08 \mathrm{dBic}$ at 


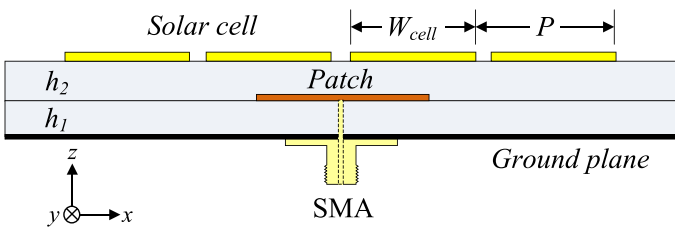

(a)

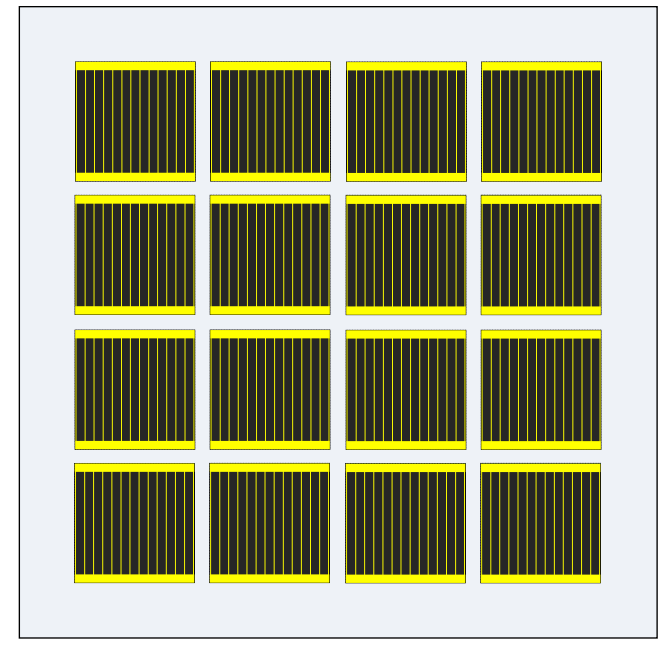

(b)

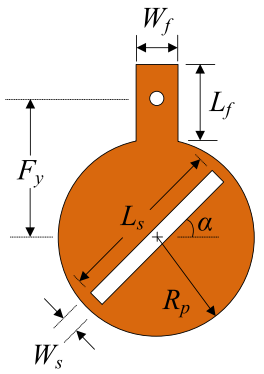

(c)

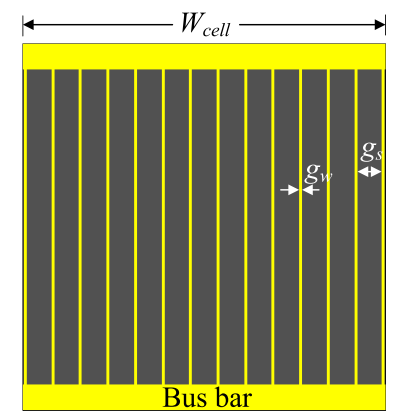

(d)
Fig. 12. Geometry of the antenna: (a) side view, (b) top view of the metasurface solar cell structure, (c) top view of the driven patch, and (d) top view of the single solar cell structure. The solar cell consisted of metal grids (bus bar and finger) on top of GaAs layers and a metallic layer for direct current contact on the bottom.

6.0 GHz. In addition, the antenna array yielded a broadside RHCP radiation with a symmetrical profile, low sidelobe level, and high radiation efficiency.

Another planar CP antenna array with a high gain and wideband operation was investigated in reference [55]. The array is based on a sequentially rotated feeding network for a set of $2 \times 2 \mathrm{CP}$ metasurface-based antennas. Figure 14 shows the geometry of the sequentially fed slot-coupled metasurface antenna array. The single element is a microstrip line-fed slot antenna, which is coupled to a metasurface having $4 \times 4$ truncated corner square, patches to produce the $\mathrm{CP}$ radiation. The wideband characteristic

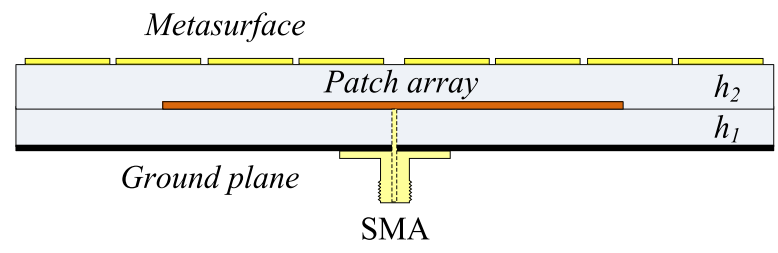

(a)

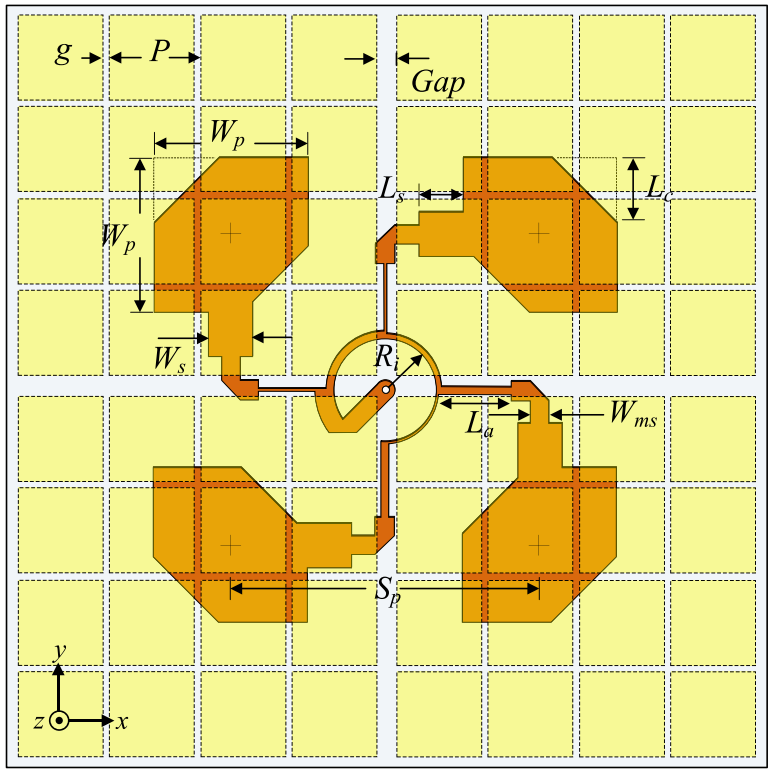

(b)

Fig. 13. Geometry of the sequentially fed metasurface antenna array: (a) side view and (b) top view. The driven patch had an offcenter position relative to the metasurface, which improves the overall antenna characteristics.

of the design is obtained by the proper combination of the feeding network and the CP metasurface-based antennas. The proposed array, with an overall size of $50 \times 50 \times$ $2.032 \mathrm{~mm}^{3}$ (approximately $1.60 \times 1.60 \times 0.065 \lambda_{o}^{3}$ at $9.6 \mathrm{GHz}$ ), was fabricated and tested. The measurements showed a $-10 \mathrm{~dB}$ impedance bandwidth of $7.88-12.0 \mathrm{GHz}$ $(41.45 \%)$ and a $3-\mathrm{dB}$ AR bandwidth of $8.4-10.6 \mathrm{GHz}$ $(23.16 \%)$. In addition, the antenna yielded broadside RHCP radiation with a peak gain of $13.5 \mathrm{dBic}$, a $3-\mathrm{dB}$ gain bandwidth of $8.6-10.8 \mathrm{GHz}(22.68 \%)$, and radiation efficiency of $>84 \%$. With these advantages and those of simple configuration, low profile, compact size, wide operating frequency, stable radiation profile, high gain, and high radiation efficiency, the proposed antenna array is a good candidate for use in wideband communication systems.

\section{Metasurface antenna at terahertz frequency}

Planar Fabry-Pérot cavity antennas have been widely investigated due to their high directivity, low complexity, and stability of fabrication [56-63]. However, these 


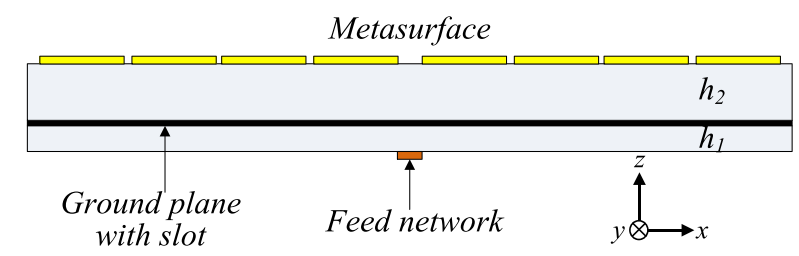

(a)

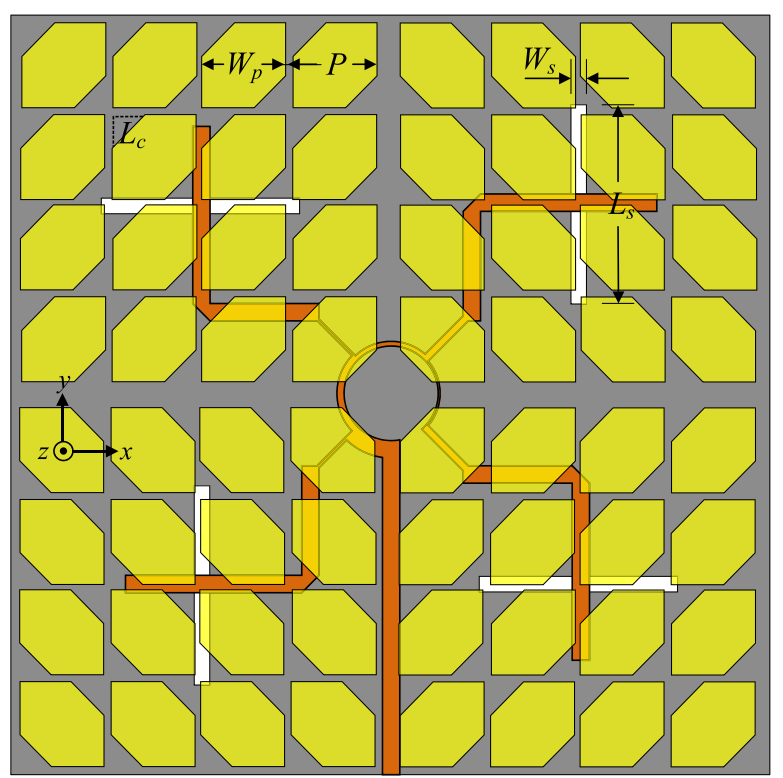

(b)

Fig. 14. Geometry of the sequentially fed slot-coupled metasurface antenna array: (a) cross-sectional view and (b) top view of the $2 \times 2$ array. The slots are etched on the ground plane, which is sandwiched between two substrates without an air gap.

antennas face design challenges regarding the improvement of their low radiation efficiency and narrow 3 -dB gain bandwidth, particularly those with electrically thick high permittivity substrates [64]. At terahertz (THz) frequencies, electrically thick substrates cause serious problems, including substrate resonance, which can be avoided by making the substrate thickness very thin with metasurface structures. This section presents antennas excited by a leaky-wave slit placed below the metasurface at a $\mathrm{THz}$ frequency.

The design of a planar, low-profile, wide-gain-bandwidth metasurface antenna at a $\mathrm{THz}$ frequency was presented in reference [65]. The geometry of the antenna for the single-feed structure is shown in Figure 15. The antenna consists of a metasurface and a planar feeding structure, both of which are patterned on an electrically thin, high-permittivity substrate $\left(\mathrm{GaAs}: \varepsilon_{\mathrm{r}}=12.9\right.$ and tan $\delta=0.006)$. The metasurface, which is printed on the top side of the substrate, consists of a periodic array of $5 \times 5$ square patches. The planar feeding structure, which is printed on the bottom side of the substrate, is a wideband leaky-wave open-ended slotline. The antenna with a single feeding structure exhibited a maximum broadside gain of

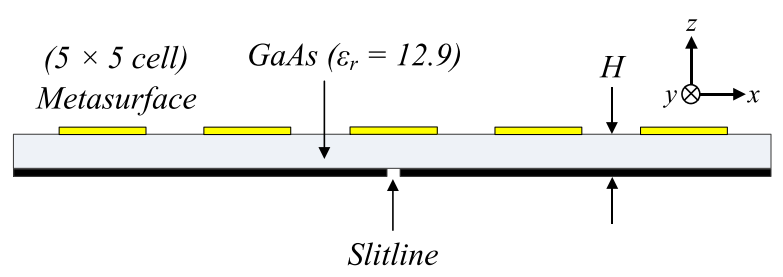

(a)

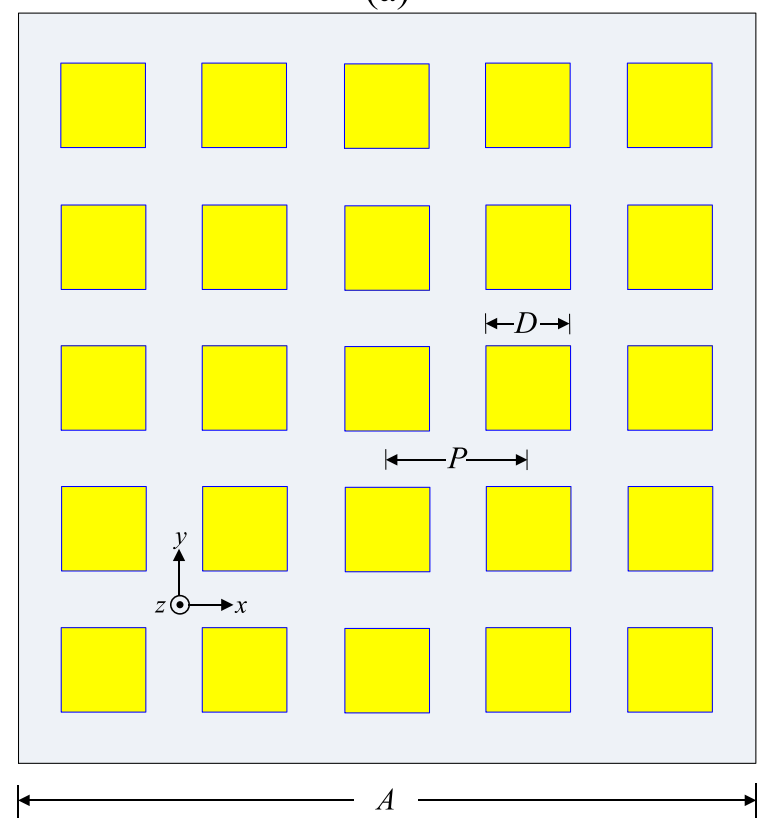

(b)

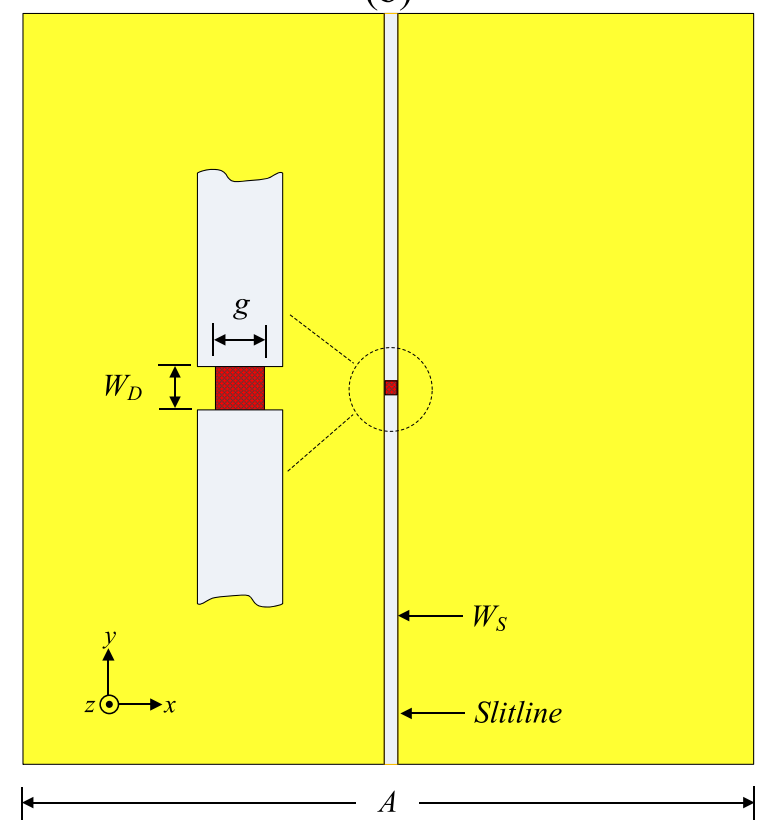

(c)

Fig. 15. Geometry of the antenna for the single feed structure: (a) side view, (b) top view of the front side, and (c) top view of the bottom side. The optimized design parameters of the $5 \times 5$ patch array antenna, which achieved the best broadside gain and a $3-\mathrm{dB}$ gain bandwidth, are $A=1800 \mu \mathrm{m}, H=40 \mu \mathrm{m}, \quad D=200 \mu \mathrm{m}$, $P=340 \mu \mathrm{m}, W_{\mathrm{S}}=15 \mu \mathrm{m}, W_{\mathrm{D}}=10 \mu \mathrm{m}$, and $g=10 \mu \mathrm{m}$. 


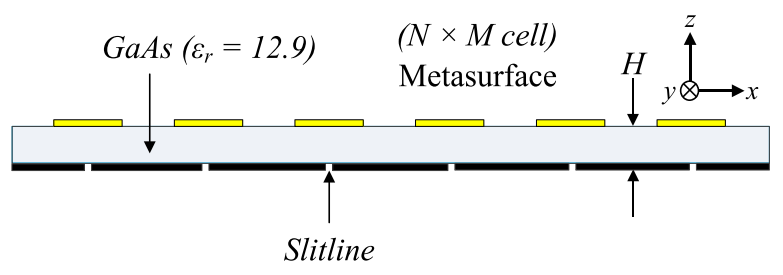

(a)

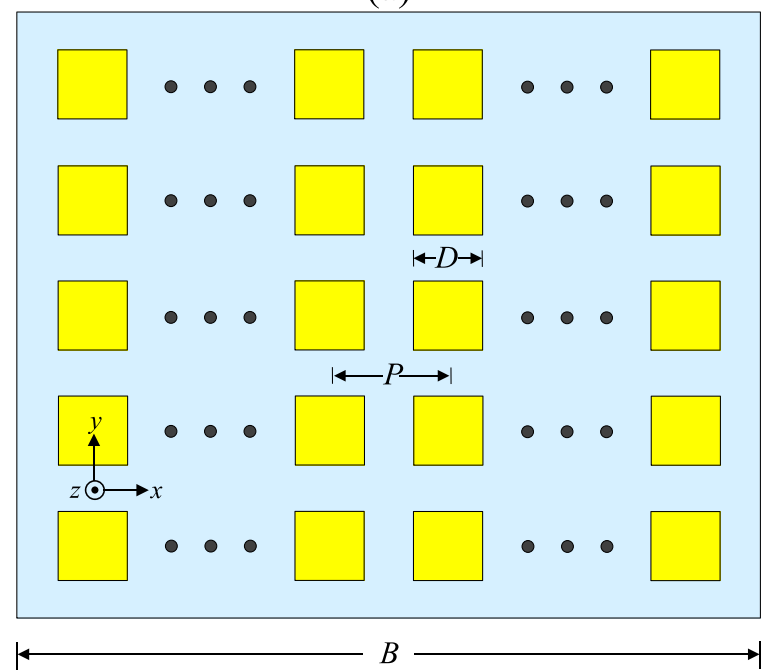

(b)

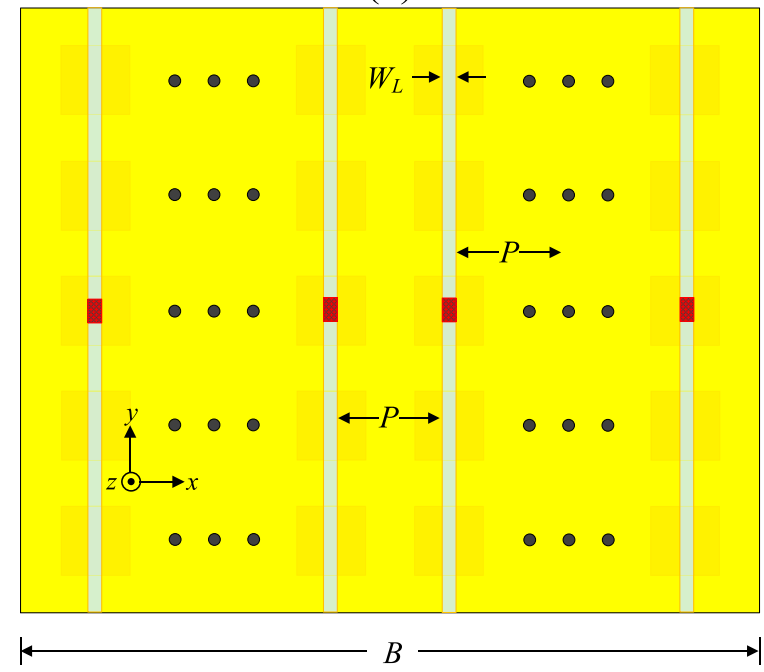

(c)

Fig. 16. Geometry of the antenna for the multiple feed structure: (a) side view, (b) top view of the front side, and (c) top view of the bottom side. The number of patches along the vertical axis ( $y$-axis) was designated as $M$, while $N$ represents the patches along the horizontal axis ( $x$-axis).

$9.8 \mathrm{dBi}$, radiation efficiency of $69 \%$, and a $3-\mathrm{dB}$ gain bandwidth of $16 \%(0.34-0.40 \mathrm{THz})$.

The antenna gain performance can be significantly improved by exciting the antenna with an array of slit feeding structures without changing the antenna size. For example, an antenna with a five-feeding structure showed a gain of $15.5 \mathrm{dBi}$, a $3-\mathrm{dB}$ gain bandwidth of $17.3 \%$, and radiation efficiency of $73 \%$. This antenna achieved a considerable size reduction in terms of device thickness in comparison to the design of a conventional lens-coupled antenna while achieving comparable gain [66]. The antenna design exhibits a low-profile, mechanical robustness, easy integration into circuit boards, and excellent low-cost mass production suitability in addition to its high-gain and widegain bandwidth characteristics.

The design and performance of low-profile, multiplefeed metasurface antennas with different numbers of patch cells and different substrate thicknesses at a $\mathrm{THz}$ frequency were presented in reference [67]. The geometry of the antenna for the multiple feed structure is shown in Figure 16. The utilized antenna designs consist of a periodic array $(N \times M)$ metallic square-patch metasurface and a planar feeding structure. The antenna gain increased linearly with an increasing number of patch cells, which were directly fed by the slit feedline. A 3-dB gain increment was observed irrespective of the substrate thickness when the number of patch cells was doubled. However, the 3-dB gain bandwidth, as well as the radiation efficiency, changed significantly with varying substrate thicknesses. The described antenna structure offers useful characteristics by means of a combination of different substrate thicknesses and patch numbers. It was shown that the antenna performed as a Fabry-Pérot cavity antenna at the high thickness substrate range, exhibiting high gain and directivity with narrow gain bandwidths, while in the low thickness substrate range, it performed as a metasurface antenna and showed wide-gain-bandwidth characteristics [68].

\section{Conclusions and future outlook}

This paper has presented a review of the designs, characteristics, and applications of various metasurfacebased antennas developed at the MCL of Ajou University. First, the metasurface antennas implemented by placing the various radiators on top of the metasurfaces were presented. This was followed by the presentation of an implementation whereby the radiator was placed below the metasurface with and without the ground plane. An interesting feature of metasurface-based antennas is their ability to generate resonances from the metasurface structure that are additional to the resonances from the radiators. The generation of these additional resonances was utilized to add operating bands or to broaden the overall antenna bandwidth. The design of a planar compact wide-gain-bandwidth metasurface antenna and its radiation characteristics were also presented at the $\mathrm{THz}$ frequency band. The $\mathrm{THz}$ antenna consists of a metasurface and a planar feeding structure, both of which are patterned on an electrically thin high-permittivity substrate. It was described how these radiators could easily be combined with different metasurface structures to achieve desired antenna characteristics. With their many outstanding performance characteristics, the metasurfacebased antennas reviewed in this article can be widely applied in many current and next generation communication systems. 
There is a plethora of applications for the design of antennas and related electromagnetic wave devices using metasurfaces. Metasurface research at the MCL will focus on one-dimensional metasurfaces and solar cell metasurface-integrated antennas. One-dimensional metasurfaces can further reduce the sizes of antenna structures and find many applications where limited spaces are required. Solar cell metasurface-integrated antennas are expected to become a highly important area of research into selfpowered antennas for sensors and Internet of Things applications.

This work was supported in part by the National Research Foundation of Korea funded by the Korea government under Grant 2018R1D1A1A02086071 and in part by the "Human Resources Program in Energy Technology" of the Korea Institute of Energy Technology Evaluation and Planning funded by the Ministry of Trade, Industry \& Energy, Republic of Korea, under Grant 20164030201380.

\section{References}

1. N. Engheta, R.W. Ziolkowski, Metamaterials: physics and engineering explorations (Wiley-Interscience, New York, 2005)

2. G.V. Eleftheriades, K.G. Balmain, Negative-refraction metamaterials (Wiley-Interscience, New York, 2005)

3. V.G. Veselago, The electrodynamics of substances with simultaneously negative values of $\varepsilon$ and $\mu$, Sov. Phys. Uspekhi 10, 509 (1968)

4. J.B. Pendry, J.A. Holden, D.J. Robbins, W.J. Stewart, Magnetism from conductors and enhanced nonlinear phenomena, IEEE Trans. Microw. Theory Tech. 47, 2075 (1999)

5. D.R. Smith, W.J. Padilla, D.C. Vier, S.C. Nemat-Nasser, S. Schultz, Composite medium with simultaneously negative permeability and permittivity, Phys. Rev. Lett. 84, 4184 (2000)

6. R.A. Shelby, D.R. Smith, S. Schultz, Experimental verification of a negative index of refraction, Science 292, 77 (2001)

7. S. Tretyakov, A. Urbas, N. Zheludev, Special issue on the history of metamaterials, J. Opt. 19, 080404 (2017)

8. A. Sihvola, Metamaterials in electromagnetics, Metamaterials 1, 2 (2007)

9. D. Sievenpiper, L. Zhang, R.F.J. Broas, N.G. Alexopoulos, E. Yablonovich, High-impedance electromagnetic surface with a forbidden frequency band, IEEE Trans. Microw. Theory Tech. 47, 2059 (1999)

10. C.A. Balanis, M.A. Amiri, A.Y. Modi, S. Pandi, C.R. Birtcher, Applications of AMC-based impedance surfaces, EPJ Appl. Metamat. 5, 1 (2018)

11. C.L. Holloway, E.F. Kuester, J.A. Gordon, J. O'Hara, J. Booth, D.R. Smith, Overview of the theory and applications of metasurfaces: the two-dimensional equivalents of metamaterials, IEEE Antennas Propag. Mag. 54, 10 (2012)

12. N. Yu, P. Genevet, M.A. Kats, F. Aieta, J.P. Tetienne, F. Capasso, Z. Gaburro, Light propagation with phase discontinuities: generalized laws of reflection and refraction, Science, 234, 333 (2011)

13. H.T. Chen, A.J. Taylor, N. Yu, A review of metasurfaces: physics and applications, Rep. Prog. Phys. 79, 076401 (2016)

14. A. Li, S. Singh, D. Sievenpiper, Metasurfaces and their applications, Nanophotonics 7, 989 (2018)
15. S. Walia, C.M. Shah, P. Gutruf, H. Nili, D.R. Chowdhury, W. Withayachumnankul, M. Bhaskaran, S. Sriram, Flexible metasurfaces and metamaterials: a review of materials and fabrication processes at micro- and nano-scales, Appl. Phys. Rev. 2, 011303 (2015)

16. F. Ding, A. Pors, S.I. Bozhevolnyi, Gradient metasurfaces: a review of fundamentals and applications, Rep. Prog. Phys. 81, 026401 (2018)

17. M. Chen, M. Kim, A.M.H. Wong, G.V. Eleftheriades, Huygens' metasurfaces from microwaves to optics: a review, Nanophotonics 7, 1207 (2018)

18. Y. Lee, S.J. Kim, H. Park, B. Lee, Metamaterials and metasurfaces for sensor applications, Sensors 17, 1726 (2017)

19. L. Huang, S. Zhang, T. Zentgraf, Metasurface holography: from fundamentals to applications, Nanophotonics 7, 1169 (2018)

20. N. Yu, F. Capasso, Flat optics with designer metasurfaces, Nat. Mat. 13, 139 (2014)

21. H.H. Hsiao, C.H. Chu, D.P. Tsai, Fundamentals and applications of metasurfaces, Small Methods 1, 1600064 (2017)

22. S.B. Glybovski, S.A. Tretyakov, P.A. Belov, Y.S. Kivshar, C.R. Simovski, Metasurfaces: from microwaves to visible, Phys. Rep. 634, 1 (2016)

23. D.J. Park, S.J. Park, I. Park, Y.H. Ahn, Dielectric substrate effect on the metamaterial resonances in terahertz frequency range, Curr. Appl. Phys. 14, 570 (2014)

24. Y. Dong, T. Itoh, Metamaterial-based antennas, Proc. IEEE 100, 2271 (2012)

25. B. Ratni, E. Bochkova, G.P. Piau, A. de Lustrac, A. Lupu, S.N. Burokur, Design and engineering of metasurfaces for high-directivity antenna and sensing applications, EPJ Appl. Metamat. 3, 1 (2016)

26. Q. Zhang, L. Si, Y. Huang, X. Lv, W. Zhu, Low-indexmetamaterial for gain enhancement of planar terahertz antenna, AIP Adv. 4, 037103 (2014)

27. I. Yoo, M.F. Imani, T. Sleasman, D.R. Smith, Efficient complementary metamaterial element for waveguide-fed metasurface antennas, Opt. Express 24, 28686 (2016)

28. Y. Huang, L. Yang, J. Li, Y. Wang, G. Wen, Polarization conversing of metasurface for the application of wideband low-profile circular polarization slot antenna, Appl. Phys. Lett. 109, 054101 (2016)

29. N. Nasimuddin, Z. N. Chen, X. Qing, Bandwidth enhancement of a single-feed circularly polarized antenna using a metasurface: metamaterial-based wideband $\mathrm{CP}$ rectangular microstrip antenna, IEEE Antennas Propag. Mag. 58, 39 (2016)

30. F. Costa, O. Luukkonen, C. R. Simovski, A. Monorchio, S. A. Tretyakov, P. de Maagt, TE surface wave resonances on high-impedance surface based antennas: analysis and modeling, IEEE Trans. Antennas Propag. 59, 3588 (2011)

31. R. Nakamura, T. Fukusako, Broadband design of circularly polarized microstrip patch antenna using artificial ground structure with rectangular unit cells, IEEE Trans. Antennas Propag. 59, 2103 (2011)

32. S.X. Ta, I. Park, Artificial magnetic conductor based circularly polarized crossed-dipole antennas - 1. AMCstructure with grounding pins, Radio Sci. 52, 630 (2017)

33. S.X. Ta, I. Park, Artificial magnetic conductor based circularly polarized crossed-dipole antennas - 2. AMCstructure without grounding pins, Radio Sci. 52, 642 (2017) 
34. A.R.I. Lamminen, A.R. Vimpari, J. Saily, UC-EBG on LTCC for $60-\mathrm{GHz}$ frequency band antenna applications, IEEE Trans. Antennas Propag. 57, 2904 (2009)

35. R. Remski, Analysis of PBG surface using Ansoft HFSS, Microw. J. 43, 190 (2000)

36. A.P. Feresidis, G. Goussetis, S. Wang, J. C. Vardaxoglou, Artificial magnetic conductor surfaces and their application to low-profile high-gain antennas, IEEE Trans. Antennas Propag. 53, 209 (2005)

37. H. Mosallaei, K. Sarabandi, Antenna miniaturization and bandwidth enhancement using a reactive impedance substrate, IEEE Trans. Antennas Propag. 52, 2403 (2004)

38. S.X. Ta, I. Park, Dual-band operation of a circularly polarized radiator on a finite artificial magnetic conductor surface, J. Electromagn. Waves Appl. 28, 880 (2014)

39. S.X. Ta, I. Park, R.W. Ziolkowski, Crossed dipole antennas: a review, IEEE Antennas Propag. Mag. 57, 107 (2015)

40. H. Huang, Y. Liu, S. Zhang, S. Gong, Multiband metamaterial-loaded monopole antenna for WLAN/WiMAX applications, IEEE Antennas Wireless Propag. Lett. 14, $662(2015)$

41. S.X. Ta, I. Park, R.W. Ziolkowski, Circularly polarized crossed dipole on an HIS for $2.4 / 5.2 / 5.8-\mathrm{GHz}$ WLAN applications, IEEE Antennas Wireless Propag. Lett. 12, $1464(2013)$

42. S. X. Ta, I. Park, Dual-band low-profile crossed asymmetric dipole antenna on dual-band AMC surface, IEEE Antennas Wireless Propag. Lett. 13, 587 (2014)

43. S.X. Ta, I. Park, Design of miniaturized dual-band artificial magnetic conductor with easy control of second/first resonant frequency ratio, J. Electromagn. Eng. Sci. 13, 104 (2013)

44. H.H. Tran, I. Park, A dual-wideband circularly polarized antenna using an artificial magnetic conductor, IEEE Antennas Wireless Propag. Lett. 15, 950 (2016)

45. H.H. Tran, I. Park, Wideband circularly polarized low-profile antenna using artificial magnetic conductor, J. Electromagn. Waves Appl. 30, 889 (2016)

46. K. Kandasamy, B. Majumder, J. Mukherjee, K.P. Ray, LowRCS and polarization-reconfigurable antenna using crossslot-based metasurface, IEEE Antennas Wireless Propag. Lett. 14, 1638 (2015)

47. K. Li, L. Li, Y. M. Cai, C. Zhu, C. H. Liang, A novel design of low-profile dual-band circularly polarized antenna with meta-surface, IEEE Antennas Wireless Propag. Lett. 14, $1650(2015)$

48. W.E.I. Liu, Z.N. Chen, X. Qing, J. Shi, F.H. Lin, Miniaturized wideband metasurface antennas, IEEE Trans. Antennas Propag. 65, 7345 (2017)

49. W. Yang, Q. Meng, W. Che, L. Gu, Q. Xue, Low-profile wideband dual-circularly polarized metasurface antenna array with large beamwidth, IEEE Antennas Wireless Propag. Lett. 17, 1613 (2018)

50. H.L. Zhu, S.W. Cheung, K.L. Chung, T.I. Yuk, Linear-tocircular polarization conversion using metasurface, IEEE Trans. Antennas Propag. 61, 4615 (2013)
51. H.L. Zhu, S.W. Cheung, X.H. Liu, T.I. Yuk, Design of polarization reconfigurable antenna using metasurface, IEEE Trans. Antennas Propag. 62, 2891 (2014)

52. S.X. Ta, I. Park, Low-profile broadband circularly polarized patch antenna using metasurface, IEEE Trans. Antennas Propag. 61, 5929 (2015)

53. S.X. Ta, I. Park, Solar-cell metasurface-integrated circularly polarized antenna with $100 \%$ insolation, IEEE Antennas Wireless Propag. Lett. 16, 2675 (2017)

54. S.X. Ta, I. Park, Compact wideband circularly polarized patch antenna array using metasurface, IEEE Antennas Wireless Propag. Lett. 16, 1932 (2017)

55. S.X. Ta, I. Park, Planar wideband circularly polarized metasurface-based antenna array, J. Electromagn. Waves Appl. 30, 1621 (2016)

56. G.V. Trentini, Partially reflecting sheet arrays, IRE Trans. Antennas Propag. 4, 666 (1956)

57. R.N. Clarke, C.B. Rosenberg, Fabry-Pérot and open resonators at microwave and millimetre wave frequencies, 2-300 GHz, J. Phys. E: Sci. Instrum. 15, 9 (1982)

58. Z. Liu, Fabry-Pérot resonator antenna, J. Infrared Milli. Terahz. Waves 31, 39 (2010)

59. C.P. Huang, C.T. Chan, Deep subwavelength Fabry-Pérot resonances, EPJ Appl. Metamat. 1, 1 (2014)

60. T.K. Nguyen, B.Q. Ta, I. Park, Design of a planar, high-gain, substrate-integrated Fabry-Pérot cavity antenna at terahertz frequency, Curr. Appl. Phys. 2015, 1047 (2015)

61. H.H. Tran, I. Park, High-gain wideband resonant cavity antenna with single half-effective-wavelength-thick dielectric layer, Microw. Opt. Technol. Lett. 58, 1693 (2016)

62. N. Hussain, T.K. Nguyen, I. Park, Performance comparison of a planar substrate-integrated Fabry-Pérot cavity antenna with different unit cells at terahertz frequency, European Conference on Antennas and Propagation (EuCAP), 1 (2016)

63. T.S. Kwon, J.G. Lee, J.H. Lee, The gain estimation of a Fabry-Perot cavity (FPC) antenna with a finite dimension, J. Electromagn. Eng. Sci. 17, 241 (2017)

64. T.K. Nguyen, T.A. Ho, I. Park, H. Han, Full-wavelength dipole antenna on a GaAs membrane covered by a frequency selective surface for a terahertz photomixer, Prog. Electromagn. Res. 131, 441 (2012)

65. N. Hussain, I. Park, Design of a wide-gain-bandwidth metasurface antenna at terahertz frequency, AIP Adv. 7, 055313 (2017)

66. T.K. Nguyen, F. Rotermund, I. Park, A traveling-wave stripline dipole antenna on a substrate lens at terahertz frequency, Curr. Appl. Phys. 14, 998 (2014)

67. N. Hussain, I. Park, Performance of multiple-feed metasurface antennas with different numbers of patch cells and different substrate thicknesses, Adv. Comp. Electromagn. J. 33, $49(2018)$

68. N. Hussain, K.E. Kedze, I. Park, Performance of a planar leaky-wave slit antenna for different values of substrate thickness, J. Electromagn. Eng. Sci. 17, 202 (2017)

Cite this article as: Ikmo Park, Application of metasurfaces in the design of performance-enhanced low-profile antennas, EPJ Appl. Metamat. 5, 11 (2018) 